\title{
Memory effects in microscopic traffic models and wide scattering in flow-density data
}

\author{
Martin Treiber and Dirk Helbing \\ Institute for Economics and Traffic, Dresden University of Technology, Andreas-Schubert-Str. 23, 01062 Dresden, Germany
}

(February 2, 2008)

\begin{abstract}
By means of microscopic simulations we show that non-instantaneous adaptation of the driving behaviour to the traffic situation together with the conventional measurement method of flowdensity data can explain the observed inverse- $\lambda$ shape and the wide scattering of flow-density data in "synchronized" congested traffic. We model a memory effect in the response of drivers to the traffic situation for a wide class of car-following models by introducing a new dynamical variable describing the adaptation of drivers to the surrounding traffic situation during the past few minutes ("subjective level of service") and couple this internal state to parameters of the underlying model that are related to the driving style. For illustration, we use the intelligent-driver model (IDM) as underlying model, characterize the level of service solely by the velocity and couple the internal variable to the IDM parameter "netto time gap", modelling an increase of the time gap in congested traffic ("frustration effect"), that is supported by single-vehicle data. We simulate open systems with a bottleneck and obtain flow-density data by implementing "virtual detectors". Both the shape, relative size and apparent "stochasticity" of the region of the scattered data points agree nearly quantitatively with empirical data. Wide scattering is even observed for identical vehicles, although the proposed model is a time-continuous, deterministic, single-lane car-following model with a unique fundamental diagram.
\end{abstract}

\section{INTRODUCTION}

The nature of "synchronized" traffic flow is one of the most controversial subjects in traffic theory $[1,2]$. It is a form of congested traffic with nonzero flows typically found upstream of inhomogeneities (e.g. freeway bottlenecks), characterized by an erratic motion of timedependent flow-density data in a two-dimensional area (and a synchronization of the time-dependent average vehicle velocities among neighboring lanes) [3-5].

The wide scattering of the data points for congested traffic seems to exclude explanations in terms of traffic models assuming a fundamental (steady-state) relation $Q_{\mathrm{e}}(\rho)$ between the flow $Q$ and the density $\rho$. In response, models with non-unique flow-density relations (or velocity-distance relations) have been proposed both on a macroscopic level [6], as car-following models [7-10], and as cellular automata [11]. The empirical data scattering has also triggered a flood of publications in physics journals with various other suggestions ranging from shock waves propagating forward or backward [3], effects of lane changing, changes in the behavior of "frustrated" drivers [12-14], anticipation effects $[15,16]$, or a trapping of vehicles [17]. Another obvious explanation of the scattering lies in the heterogeneity of vehicles (such as cars and trucks) and driving styles (such as defensive or aggressive) on any real road [18]. In fact, statistical analyses of single-vehicle data show a particularly wide scattering of the time gaps between successive vehicles in congested traffic [19-21]. Furthermore, the average netto time gaps increase in congested traffic suggesting that "frustration effects" are real [21]. Macroscopic simulations taking into account observed variations in the truck percentage [22] or direct microsimulations with two types of vehicles [23] could explain a great deal of the observed scattering, but the 2D-regions remained somewhat smaller than in the observed data.

Another factor possibly contributing to the wide scattering are traffic instabilities resulting in so-called "oscillating congested traffic" which is the most common form of congested traffic [24]. If the sampling time interval for data aggregation is not commensurable with the frequency of the oscillations or if the oscillations are nonperiodic, then the data points will display artificial "erratic scattering". In this case, the origin of the scattering is the method of data aggregation in combination with the conventional interpretation of flow-density data [18].

In this paper we show by means of simulations that the adaptation of drivers to the surrounding traffic on time scales of a few minutes ("memory effect") in conjunction with traffic instabilities can quantitatively explain the observed scattering. Our model is based on the observation that, after being stuck for some time in congested traffic, most drivers adapt their driving style, e.g., by increasing their preferred netto (bumper-to-bumper) time gap $T$ to the preceding vehicle [19-21,2]. Apart from congestion, other aspects of the traffic environment such as driving in the dark or in tunnels affect the driving behaviour as well [25], but will not considered in this paper.

Models based on memory effects have been successfully applied in several fields of statistical and interdisciplinary physics such as liquid crystals and polymers [26] or in reaction-diffusion systems [27]. Nonlinear Markow equations with memory Kernel have been applied to multiagent systems and financial markets and provide a statistical mechanism for the observed clustered volatility 
[28].

In the next section, we formulate the adaptation of the driving style to the surrounding traffic and incorporate the memory effects into the intelligent-driver model (IDM) [24] resulting in the IDMM ("intelligent-driver model with memory"). Like the IDM, the IDMM is a deterministic time-continuous car-following model with a unique steady-state flow-density relation.

In Section III, we presents related simulations and compare the measurements of "virtual detectors" with empirically measured traffic data. We will find a semiquantitative agreement. While the scattering is even observed in the original IDM, the memory effect is necessary to obtain the inverse- $\lambda$ shape and the correct relative size of the twodimensional region of data scattering.

In the concluding Section IV we discuss the remarkable fact that erratic scattering can be obtained from deterministic single-lane models with a unique fundamental diagram in simulations without any element of stochasticity or heterogeneity.

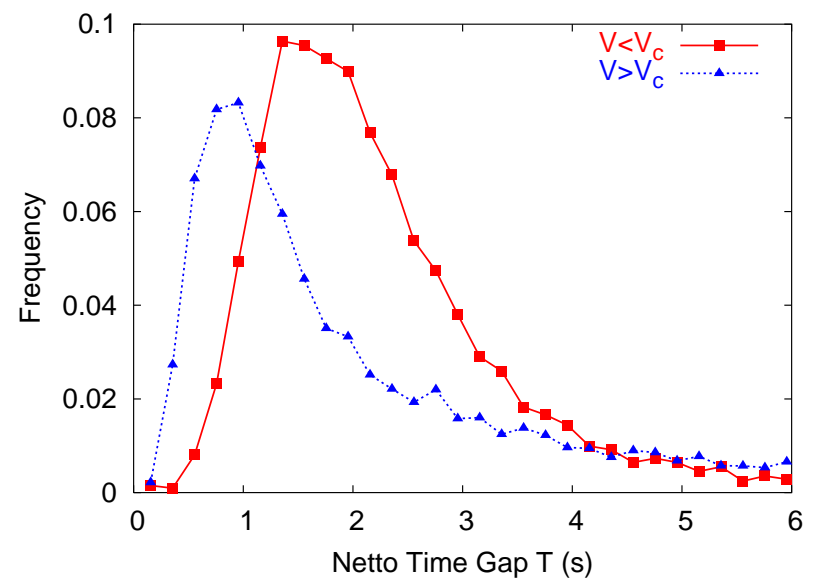

FIG. 1. Distribution of netto time headways on the left lane of the Dutch A9 from Haarlem to Amsterdam for free traffic $\left(v>v_{c}=60 \mathrm{~km} / \mathrm{h}\right)$ and congested traffic $\left(v<v_{c}\right)$.

\section{MODEL EQUATIONS}

We will formulate the memory effect in human driver behavior in a way that directly connects to existing carfollowing models. Any model can be used, the parameters of which can be interpreted in terms of the driving behaviour. The model should allow to define some desired velocity $v_{0}$ and to influence the minimum netto time gap $T$ by varying one or more of its model parameters. The outcome, of course, will depend on the details of the model used. In this paper, we apply the intelligent-driver model (IDM) [24] as underlying model, where $v_{0}$ and $T$ are model parameters themselves.

\section{A. The intelligent-driver model}

In the IDM, the acceleration of each vehicle $\alpha$ is assumed to be a continuous function of the velocity $v_{\alpha}$, the netto distance gap $s_{\alpha}$, and the velocity difference (approaching rate) $\Delta v_{\alpha}$ to the leading vehicle:

$$
\dot{v}_{\alpha}=a\left[1-\left(\frac{v_{\alpha}}{v_{0}}\right)^{4}-\left(\frac{s^{*}\left(v_{\alpha}, \Delta v_{\alpha}\right)}{s_{\alpha}}\right)^{2}\right] .
$$

This expression is an interpolation of the tendency to accelerate with $a_{f}(v):=a\left[1-\left(v / v_{0}\right)^{4}\right]$ on a free road and the tendency to brake with deceleration $-b_{\text {int }}(s, v, \Delta v):=-a\left(s^{*} / s\right)^{2}$, when vehicle $\alpha$ comes too close to the vehicle in front. The deceleration term depends on the ratio between the effective "desired minimum gap" $s^{*}$ and the actual gap $s_{\alpha}$, where the desired gap

$$
s^{*}(v, \Delta v)=s_{0}+v T+\frac{v \Delta v}{2 \sqrt{a b}}
$$

is dynamically varying with the velocity. The first term $s_{0}$ on the right-hand side denotes the small minimum distance kept in standing traffic. The second term corresponds to following the preceding vehicle with a constant "safety" netto time gap $T$. The third term is only active in non-stationary traffic and implements an accident-free "intelligent" driving behaviour including a braking strategy that, in nearly all situations, limits braking decelerations to the "comfortable deceleration" $b$.

\section{B. Adaptation of the driving style and memory effect}

We assume that all adaptations of the driving style are controlled by a single internal dynamical variable $\lambda_{\alpha}(t)$ ("subjective level of service"), which can take on values between 0 (in standing traffic) and 1 (on a free road), and that it relaxes to the instantaneous level of service $\lambda_{0}(v)$ with a relaxation time $\tau$ according to

$$
\frac{d \lambda_{\alpha}}{d t}=\frac{\lambda_{\alpha}-\lambda_{0}\left(v_{\alpha}\right)}{\tau}
$$

This means, for each driver the subjective level of service is given by the exponential moving average (EMA) of the instantaneous level of service experienced in the past:

$$
\lambda_{\alpha}(t)=\left\langle\lambda_{0 \alpha}\right\rangle_{\mathrm{EMA}}=\int_{0}^{t} \lambda_{0}\left(v_{\alpha}\left(t^{\prime}\right)\right) e^{-\left(t-t^{\prime}\right) / \tau} d t^{\prime} .
$$

We have assumed the instantaneous level of service $\lambda_{0}(v)$ to be a function of the actual velocity $v(t)$. Obviously, $\lambda_{0}(v)$ is a monotonuously increasing function with $\lambda_{0}(0)=0$ and $\lambda_{0}\left(v_{0}\right)=1$. In this paper, we specify the 
most simple "level-of-service function" satisfying these conditions:

$$
\lambda_{0}(v)=\frac{v}{v_{0}}
$$

Notice that this equation reflects the level of service or efficiency of movement from the driver's point of view, with $\lambda_{0}=1$ meaning zero hindrance and $\lambda_{0}=0$ meaning maximum hindrance. If one models heterogeneous traffic where different drivers have different desired velocities there is no "objective" level of service, only an average one.

Having defined how the traffic environment influences the degree of adaptation $\lambda_{\alpha}$ of each driver, we now specify how this internal variable influences the driving behaviour. A behavioural variable that is both measurable and strongly influencing the traffic dynamics is the netto time gap $T$. Figure 1 shows that, in congested traffic, the whole distribution of time gaps is shifted to the right compared to data of free traffic [21]. We model this by varying the corresponding IDM parameter in the range between $T_{0}$ (free traffic) and $T_{\text {jam }}=\beta_{T} T_{0}$ (traffic jam) according to

$$
T(\lambda)=T_{0}\left[\beta_{T}+\lambda\left(1-\beta_{T}\right)\right] .
$$

Herein, the adaptation factor $\beta_{T}$ is a model parameter (cf. Table I). Notice that probably other parameters of the driving style are influenced as well, such as the acceleration, the comfortable deceleration, or the desired velocity. This could be implemented by analogous equations for $a, b$, and $v_{0}$, respectively. For simplicity (and in order to have an empirically testable model), we will only consider the influence on $T$.

\begin{tabular}{l|l}
\hline \hline Parameter & Typical value \\
\hline Desired velocity $v_{0}$ & $120 \mathrm{~km} / \mathrm{h}$ \\
Netto time gap $T_{0}$ & $0.85 \mathrm{~s}$ \\
Maximum acceleration $a$ & $0.8 \mathrm{~m} / \mathrm{s}^{2}$ \\
Comfortable deceleration $b$ & $1.8 \mathrm{~m} / \mathrm{s}^{2}$ \\
Minimum distance $s_{0}$ & $1.6 \mathrm{~m}$ \\
Vehicle length $l=1 / \rho_{\max }$ & $6 \mathrm{~m}$ \\
Adaptation factor $\beta_{T}=T_{\mathrm{jam}} / T_{0}$ & 1.8 \\
Adaptation time $\tau$ & $600 \mathrm{~s}$ \\
\hline \hline
\end{tabular}

TABLE I. Model parameters of the IDMM with the values used throughout this paper. All eight model parameters have a clear vehicle- or driver-related meaning and can be determined from empirical traffic data. Note that other models recently proposed to describe similar empirical observations consist of up to 10 equations with about 20 parameters [9].
In summary, the IDMM is described by the IDM equations (1) and (2), by Eq. (6) describing how the subjective level of service $\lambda$ influences the time gap, and by the dynamical equation for the internal state itself, which can be written as

$$
\frac{d \lambda_{\alpha}}{d t}=\frac{\lambda_{\alpha}-v_{\alpha} / v_{0}}{\tau}
$$

All IDMM parameters are intuitive and can be determined from traffic data. In the special case $\beta_{T}=1$, the IDMM reverts to the original IDM. The special case $\tau=0$ corresponds to a slightly modified IDM, where the parameter $T$ in Eq. (2) is replaced by $T(v)=$ $T_{0}\left[\beta_{T}+\frac{v}{v_{0}}\left(1-\beta_{T}\right)\right]$. Table I gives the values that we will used throughout the rest of this paper unless stated otherwise.

Notice that the IDMM belongs to the class of models with a unique stationarity relation. Its steady-state following distance as a function of the velocity is given by

$$
s_{e}(v)=\frac{s_{0}+v T_{0}\left(\beta_{T}+\left(1-\beta_{T}\right) \frac{v}{v_{0}}\right)}{\sqrt{1-\left(\frac{v}{v_{0}}\right)^{4}}} .
$$

Figure 2 shows the resulting fundamental diagram for identical vehicle types for the IDMM in comparison with that of the IDM.

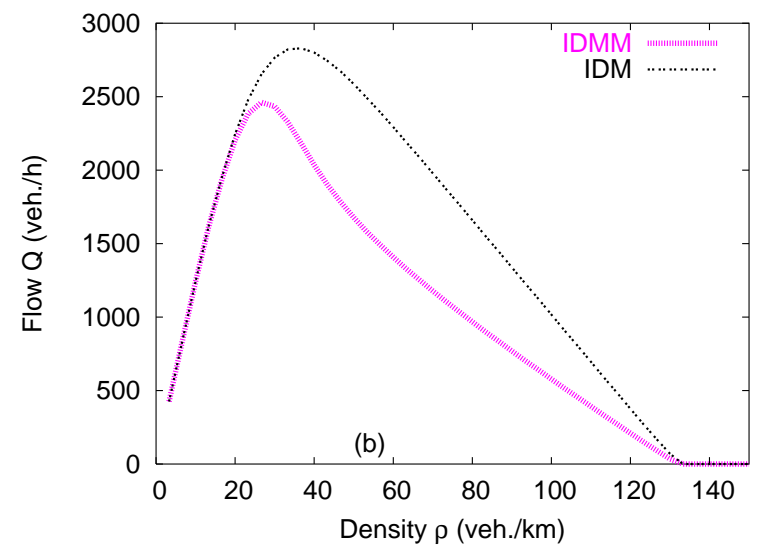

FIG. 2. Comparison of the theoretical fundamental diagrams of the IDM and IDMM.

\section{SIMULATIONS}

We have simulated a $20 \mathrm{~km}$ long road section with a bottleneck and open boundaries, assuming identical vehicles of length $l=6 \mathrm{~m}$ whose drivers behave according to the IDMM with the parameters given in Table I. The simulations have been started with very light traffic correspondig to a homogeneous density of 2 vehicles $/ \mathrm{km}$ 
and an initial velocity of $100 \mathrm{~km} / \mathrm{h}$. During the simulated time interval of 3 hours, we have simulated idealized rush-hour conditions by increasing the inflow at the upstream boundary linearly from $200 \mathrm{veh}$./h at $t=0$ to $2400 \mathrm{veh}$./h at $t=25 \mathrm{~min}$. Afterwards, the flow has been decreased linearly to $100 \mathrm{veh} . / \mathrm{h}$ at $t=180 \mathrm{~min}$. All vehicles have been initialized with $\lambda=1$, i.e., with a "memory" of free traffic. As in macroscopic traffic simulations of open systems [29], the velocity of the inflowing traffic turned out to be irrelevant, since it quickly approached the value corresponding to the "free" branch of the velocity-flow relation $v_{e}(Q)$ with a flow $Q(t)$ equal to that imposed at the boundary.

We have implemented a flow-conserving bottleneck by locally increasing the IDM parameter $T_{0}$,

$$
T_{0}=T_{0}(x)=\left\{\begin{array}{l}
1.20 \mathrm{~s} \quad 17 \mathrm{~km} \leq x<18 \mathrm{~km}, \\
0.85 \mathrm{~s} \text { otherwise }
\end{array}\right.
$$

This corresponds to lowering the road capacity and can represent any bottleneck which is not an on- or off-ramp [30]. Notice that, at the bottleneck, this means that the actual time headway $T$, as specified by Eq. (6) with (9), depends both directly on $x$ and on the subjective level of service of the driver resulting in another indirect dependence on $x$.

The simulation has been performed using an explicit integration scheme. Output is produced by implementing "virtual detectors" at $x=9 \mathrm{~km}$ and $x=12 \mathrm{~km}$, with data aggregation periods of $T_{\text {aggr }}=60 \mathrm{~s}$. In each aggregation interval $i$, the traffic flow

$$
Q_{i}=n_{i} / T_{\text {aggr }}
$$

is determined by counting the number $n_{i}$ of crossing vehicles, the average velocity $V_{i}$ is calculated by the arithmetic average

$$
V_{i}=\frac{1}{n_{i}} \sum_{\alpha=1}^{n_{i}} v_{\alpha}
$$

and the density by

$$
\rho_{i}=Q_{i} / V_{i},
$$

as in many practical cases.
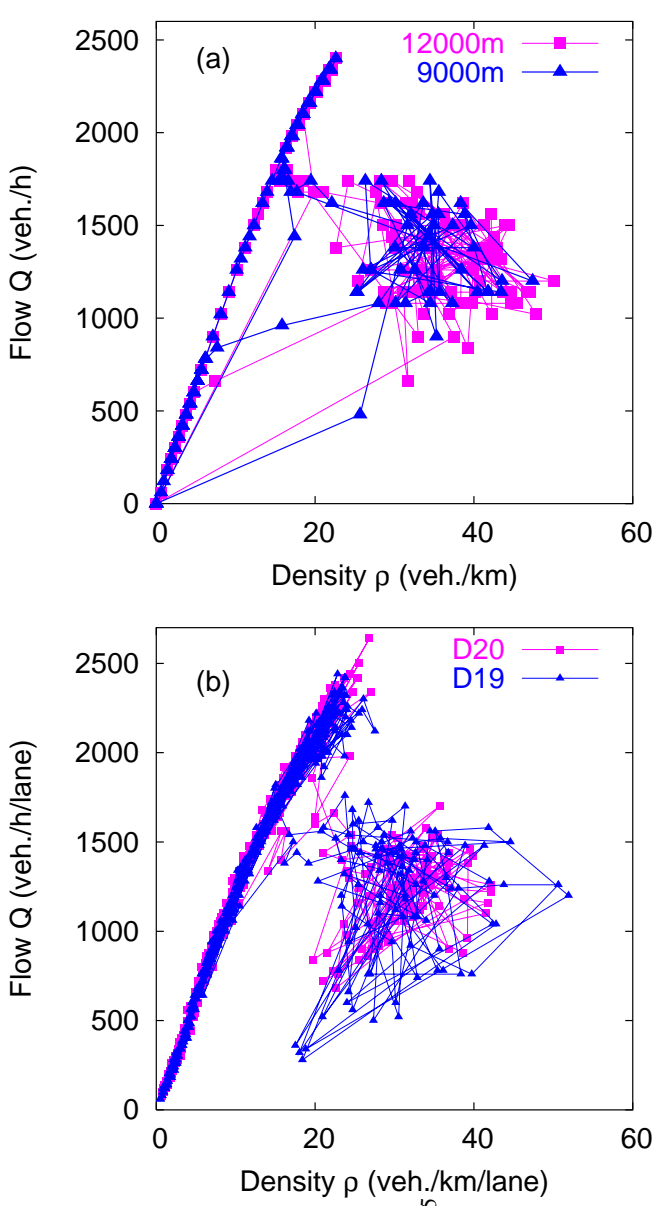

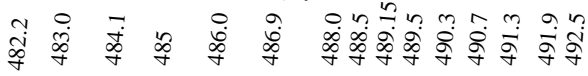

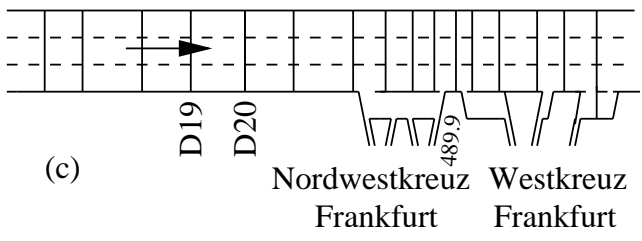

FIG. 3. (a) Simulated flow-density data of two virtual detectors compared with (b) flow-density data from the German freeway A9-South near Frankfurt on July 31, 2001. (c) Location of the detectors for the empirical data. Here, the intersection "Nordwestkreuz" serves as bottleneck.

Figure 3 displays the resulting flow-density data of the two virtual detectors, compared with empirical data from real traffic. Both diagrams shows (i) the characteristic wide and erratic scattering of the data points which is a signature of "synchronized traffic", (ii) the characteristic inverse- $\lambda$ shape with a maximum $Q_{\max }$ of traffic flow in free traffic (immediately prior to the breakdown), which is distinctively higher than the typical flows in the congested traffic $Q_{\mathrm{CT}}$ after breakdown. 


\section{A. Interpretation of macroscopic traffic data}

The question arises what causes the obvious stochasticity in the data of the virtual detectors although everything in the simulation is deterministic (including the upstream boundary condition and the implementation of the bottleneck), although all drivers and vehicles are treated identically, and not even lane changes may serve as possible source for fluctuations. The only possible source of the fluctuations are traffic instabilities which we will analyse in the following.
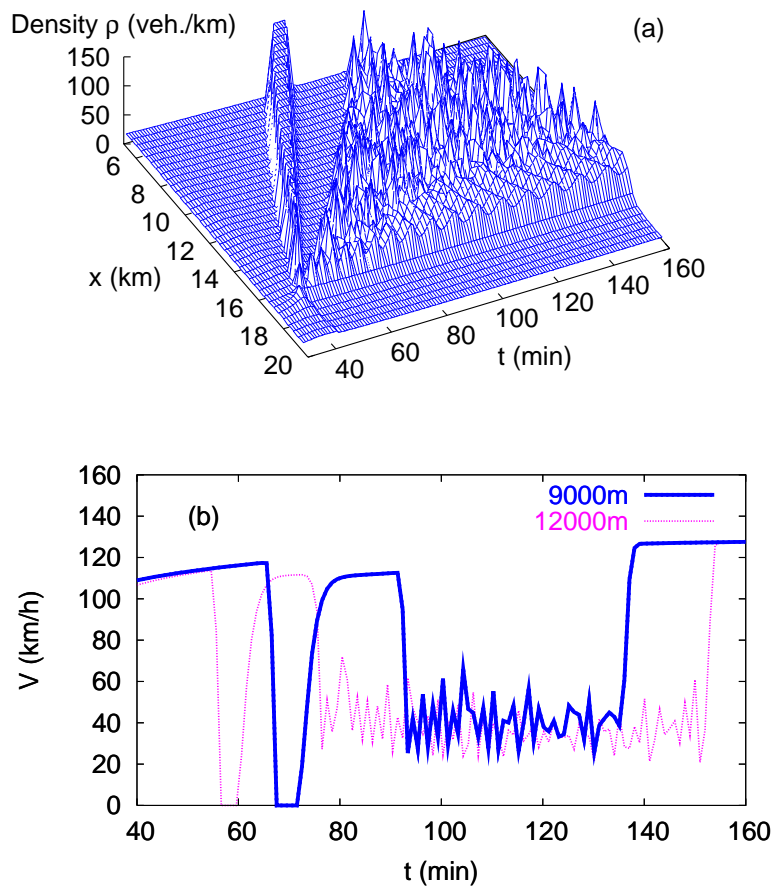

FIG. 4. (a) Spatio-temporal dynamics of the traffic density and (b) time series of the velocity at the two virtual detectors of the simulation shown in Fig. 3.

Figure 4(a) shows that at about $t=40 \mathrm{~min}$, a traffic breakdown occurs near the bottleneck at $x=12 \mathrm{~km}$, triggering one isolated wide jam with zero flow and a region of congested traffic with nonzero flow upstream of the bottleneck. In the congested region, initially small oscillations behind the bottleneck (oscillating congested traffic, OCT) increase their amplitude while travelling further upstream, and finally some isolated stop-and-go waves (called "wide moving jams" in [31]) are emitted from the congested region travelling further upstream (triggered stop-and-go traffic [32]). The time series of the virtual detectors (see Fig. 4(b)) show that the wide jam also crosses the detectors. Since the jam and the oscillations of the OCT state are the only possible sources of fluctuations, the scattering in the data of the virtual detectors obviously can be traced back to longitudinal instabilities in connection with the interpretation of the macroscopic data.

Based on theoretical investigations [3], one might expect a "jam line" in the flow-density diagram of Fig. 3 stemming from the wide jam crossing the virtual detectors. However, the jam line is missing. Moreover, the highest "measured" density is only about 50 veh. $/ \mathrm{km}$, although the model parameters (Table I) imply a jam density of at least $\rho_{\mathrm{jam}}=1 /\left(l+s_{0}\right)=130 \mathrm{veh} . / \mathrm{km}$.

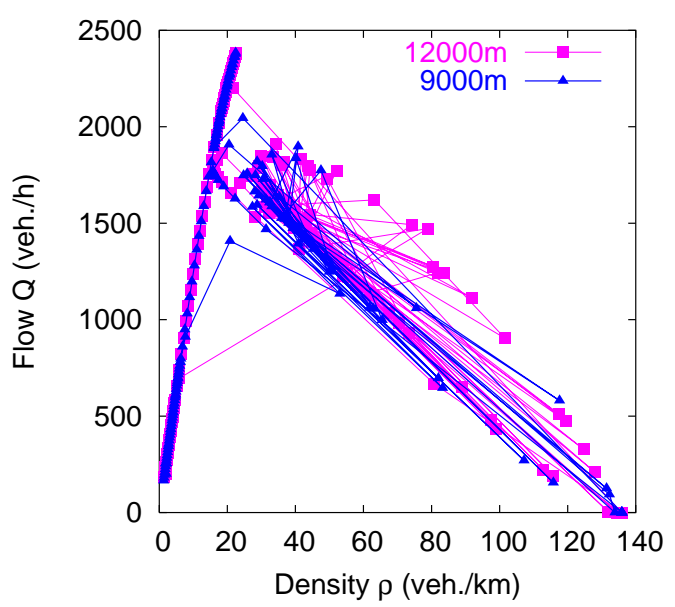

FIG. 5. Flow-density plot as in Fig. 3, but using no data aggregation and the real density (13).

To check, whether this is an artefact of the data interpretation, in Fig. 5 we have plotted the flow-density diagram of the same simulation at the same locations, but this time using the true spatial density

$$
\rho(x, t)=\frac{1}{x_{\alpha-1}(t)-x_{\alpha}(t)}, \quad x_{\alpha} \leq x<x_{\alpha-1},
$$

which one would obtain by "snapshots" at fixed times rather than the macroscopic density (12). In the data for $x=9 \mathrm{~km}$, one clearly sees the signatures of the fully developed jam in form of a straight "jam line" $J$, connecting the points $\left(\rho_{\text {jam }}, Q_{\text {jam }}\right)=(130 / \mathrm{km}, 0)$ and $\left(\rho_{\text {out }}, Q_{\text {out }}\right)=$ (18 veh./km, 1750 veh./h). Notice that, in accordance with observations [31], the outflow from isolated jams is distinctively lower than $Q_{\max }$. Moreover, the data at both locations show more than one instance of zero or nearly zero flow at densities near the maximum density, which can be seen neither in the flow-density data nor in the time series of the velocity of the virtual detectors, cf. Fig. 4. The reason is that the virtual detectors display finite velocities and flows whenever at least one car crosses the detector during the sampling time interval. Thus, periods of standing traffic of up to the double sampling time, i.e., up to 2 minutes, may not be observed in the detector output.

If we assume that the simulation captures some essential aspects of real traffic we conclude that (i) a jam 
line probably exists in real traffic but cannot be found in flow-density data of stationary detectors, (ii) when looking only at aggregated traffic data, one might get a wrong picture of the actual traffic dynamics.

\section{B. Analysis of the adaptation effect}

We now proceed to investigate the effects of the new IDMM parameters. We have simulated the same system of equations with various values of the adaptation factor $\beta_{T}$ and the adaptation time $\tau$. It turned out that both the values of $Q_{\text {out }}$ and $Q_{\mathrm{CT}}$ decrease with $\beta_{T}$, while $Q_{\max }$ essentially remains unchanged. This is plausible, since $\left(\beta_{T}-1\right)$ describes the strength of the "frustration effect" after driving in congested traffic for some time, while the value of $Q_{\max }$ is related to free traffic where frustration effects play a minor role. Furthermore, $Q_{\mathrm{CT}}$ decreases with $\tau$. Since the time spent in congestion behind bottlenecks is typically of the order of the adaptation time or longer, drivers are adapted to congested traffic when they get closer to the downstream front of the congestion area near the bottleneck. Consequently, it takes some time to revert to the more "aggressive" driving style in free traffic, explaining the decrease of $Q_{\text {out }}$ with $\tau$.

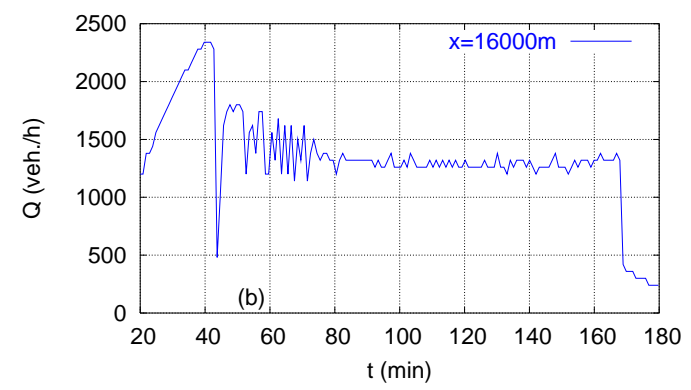

FIG. 6. Time series of traffic flow for the simulation of Fig. 4 , measured by a virtual detector at $x=11000 \mathrm{~m}$ near the bottleneck. Notice that the flow $Q_{\text {out }} \approx 1750$ veh./h in the interval $40 \mathrm{~min} \leq t \leq 50 \mathrm{~min}$ is related to the outflow from the isolated jam and not the bottleneck.

One might argue that the drivers should adapt instantaneously to the traffic situation. There is empirical evidence, however, that the characteristic time scale of the adaptation is not negligible [2]: In data of congested traffic measured near the bottleneck causing the breakdown, one often observes that, after the initial drop of the traffic flow, the flow decreases further during the first 10 or 20 minutes after the breakdown, cf. e.g., Fig. 12 in [24]. However, according to traffic theory, the outflow from jams and thus the measured flow near the bottleneck are constant for fixed driving styles. Assuming that, after the breakdown, the length of the congested area behind the bottleneck and thus the waiting time of each driver increases, gradual adaptations naturally explain the ob- servations. Figure 6 shows this effect in the simulated measurement of a virtual detector near the downstream congestion front. The average flow is highest in the beginning (at $t=60 \mathrm{~min}$ ) and at the end (at $t=160 \mathrm{~min}$ ) of the congestion. However, it eventually decreases from $Q_{\mathrm{CT}} \approx 1500 \mathrm{veh}$. $/ \mathrm{h}$ to $Q_{\mathrm{CT}} \approx 1300 \mathrm{veh}$. $/ \mathrm{h}$ at $t=120 \mathrm{~min}$, where the length of the congestion reaches its maximum value of about $10 \mathrm{~km}$.

\section{Comparison with the IDM}

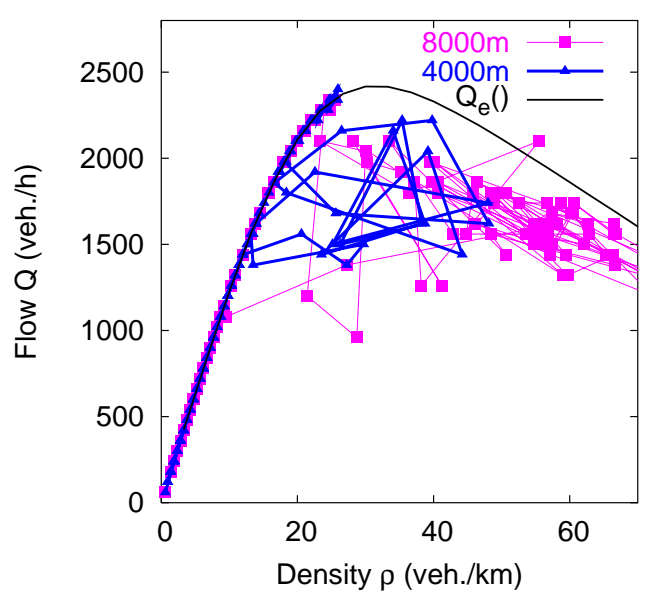

FIG. 7. Flow-density plot as in Fig. 3, simulated with the IDM without memory effects. To obtain the same degree of capacity and stability, the parameters $T$ and $a$ have been changed to $1.05 \mathrm{~s}$ and $1 \mathrm{~m} / \mathrm{s}^{2}$, respectively.

The question arises to which extent (i) the wide scattering, (ii) the distinct hysteresis effects indicated by the ratios $Q_{\max } / Q_{\mathrm{CT}}$ and $Q_{\max } / Q_{\text {jam }}$, and (iii) the low values of the "measured" densities in congested and jammed traffic are new features of the IDMM or occur in the original IDM without memory effects as well. Figure 7 shows a simulation with the original IDM, which is a special case of the IDMM for $\beta_{T}=1$. The virtual detectors display scattering as well. However, the hysteresis effects are much smaller and the density of congested traffic is shifted to higher values, which are, in particular, higher than the values usually observed in empirical data.

\section{DISCUSSION}

We have modeled a memory effect in the behavior of drivers by coupling existing car-following models to dynamical equations for some model parameters such as the minimum (safe) time gap, the desired velocity, or the typical acceleration. In this paper, we have used the IDM as underlying model resulting in the IDMM, the "IDM 
with memory". The Gipps model [33] seems to be a suitable candidate as well. It should be straightforward to apply the same concept to macroscopic models such as the GKT [34,14] and to cellular automata. In fact, the slow-to-start rule [35] can be interpreted as the special case of an instantaneous adaptation $(\tau=0)$, which is only effective for standing traffic, i.e., the corresponding "level-of-service function", Eq. (5), would be given by $\lambda_{0}(v)=1$ for $v>0$ and $\lambda_{0}(v)=0$ for $v=0$.

The concept could be also generalized to include the traffic density or the velocity variance in determining the level-of-service function. This would allow to model different kinds of adaptation behavior to different types of congested traffic such as homogeneous congested traffic (HCT) and oscillating congested traffic (OCT) [32].

As is the case for the IDM, the IDMM is a deterministic car-following model with a unique fundamental diagram. It has two new parameters, the adaptation factor $\beta_{T}$ and the adaptation time $\tau$, which can be estimated from single-vehicle data. Simulations with the IDMM suggest that the adaptation of drivers to the surrounding traffic happens on time scales of a few minutes and play, in fact, an important role in explaining the inverse- $\lambda$ shape and the wide scattering of flow-density data in the congested regime measured by stationary detectors. Despite its simplicity, the model seems to be accurate enough to enable, for the first time, a direct analysis of the conventional interpretation of macroscopic flow-density data with surprising results both for the theoretician and the practitioner.

For the theoretician, probably the most interesting result is the direct demonstration of a simple mechanism that explains the much-discussed wide scattering of congested traffic by longitudinal traffic instabilities. Important for the practitioner, the results suggest that the real traffic situation, in terms of the traffic density, is often worse than the macroscopic density data suggest. The resulting oscillations and even short periods of standing traffic are hidden in the "scattering" of the data. In contrast to previous results guided by theoretical considerations [5] the simulations suggest that congested traffic is nearly always unstable. This is supported by our analysis of more than 300 empirical examples of congestion from various freeways with a new visualization tool [36]. The majority of all congested traffic patterns displayed stopand-go waves, many of them with growing amplitudes while they travel upstream. Another factor obscuring the instability of real congested traffic is the often observed convective stability, i.e., growing perturbations can only propagate upstream, resulting in homogeneous congested traffic of high density near the downstream front of congestion ("pinch effect").

In our simulations, we have excluded most sources that would not surprise to produce scattering: We have not assumed heterogeneous multi-lane traffic exposed to fluctuation effects. Instead, we have assumed identical vehi- cles on a single lane with a dynamics given by a deterministic model with a unique fundamental diagram. We do not claim, however, that longitudinal instabilities and the memory effect would be the only cause leading to the observed scattering. Obviously, heterogeneous traffic plays a role as well. Furthermore, the role of lane changes remains to be investigated. Finally, it should be emphasized that we have investigated macroscopic implications of a microscopic model. To explain microscopic statistical properties such as the observed scaling law for the fluctuations of sample-average time headways [21], one probably needs to simulate both heterogeneous and multi-lane traffic. Microscopic statistical properties will be investigated in a forth-coming paper.

Acknowledgments: The authors would like to thank for financial support by the DFG (grant No. He 2789) and Arne Kesting for providing Fig. 1. We are also grateful to the Dutch Ministry of Transport, Public Works and Water Management for providing the single-vehicle induction-loop-detector data.

[1] D. Helbing, Review of Modern Physics 73, 1067 (2001).

[2] M. Fukui, Y. Sugiyama, M. Schreckenberg, and D. E. Wolf (eds.), Traffic and Granular Flow '01: Social, Traffic, and Granular Dynamics (Springer, Berlin, 2003).

[3] B. Kerner and H. Rehborn, Phys. Rev. E 53, R4275 (1996).

[4] B. Kerner and H. Rehborn, Phys. Rev. Lett. 79, 4030 (1997).

[5] B. Kerner, Phys. Rev. E 65, 046138 (2002).

[6] P. Nelson, Phys. Rev. E 61, 383 (2000).

[7] R. Wiedemann, Simulation des Straßenverkehrsflusses, Schriftenreihe des IfV Vol. 8, Institut für Verkehrswesen, Universität Karlsruhe (1974).

[8] A. Namazi, N. Eissfeldt, and A. Schadschneider, Europ. Journal of Phys. B30, 559 (2002).

[9] B. S. Kerner and S. L. Klenov, J. Phys. A: Math. Gen. 35, L31 (2002).

[10] D. Helbing, D. Batic, M. Schönhof, and M. Treiber, Physica A 303, 251 (2002).

[11] B. S. Kerner, S. L. Klenov, and D. Wolf, J. Phys. A: Math. Gen. 35, 9971 (2002).

[12] W. Brilon and M. Ponzlet, in Traffic and Granular Flow, edited by D. E. Wolf, M. Schreckenberg, and A. Bachem (World Scientific, Singapore, 1996), pp. 23-40.

[13] S. Krauß, Microscopic Modeling of Traffic Flow: Investigation of Collision Free Vehicle Dynamics (DLR, Raumfahrt e.V., Cologne, 1998), Report No. 98-08.

[14] M. Treiber and D. Helbing, Explanation of observed features of self-organization in traffic flow, e-print condmat/9901239.

[15] H. Lenz, C. Wagner, and R. Sollacher, European Physical 
Journal B7, 331 (1998).

[16] W. Knospe, L. Santen, A. Schadschneider, and M. Schreckenberg, Phys. Rev. E 65, 015101 (2001).

[17] I. Lubashevsky, R. Mahnke, P. Wagner, and S. Kalenkov, Phys. Rev. E 66, 016117 (2002).

[18] J. H. Banks, An Investigation of some Characteristics of Congested Flow, 1998, J. H. Banks, An Investigation of some Characteristics of Congested Flow, Transportation Research Board, submitted 1998.

[19] B. Tilch and D. Helbing, in Traffic and Granular Flow '99, edited by D. Helbing, H. Herrmann, M. Schreckenberg, and D. Wolf (Springer, Berlin, 2000), pp. 333-338.

[20] L. Neubert, L. Santen, A. Schadschneider, and M. Schreckenberg, in Traffic and Granular Flow '99, edited by D. Helbing, H. Herrmann, M. Schreckenberg, and D. Wolf (Springer, Berlin, 2000), pp. 307-314.

[21] K. Nishinari, M. Treiber and D. Helbing, Explaining the wide scattering of synchronized traffic data by time gap statistics, cond-mat/0212295 (2002).

[22] M. Treiber and D. Helbing, J. Phys. A 32, L17 (1999).

[23] M. Treiber, A. Hennecke, and D. Helbing, in Traffic and Granular Flow '99, edited by D. Helbing, H. Herrmann, M. Schreckenberg, and D. Wolf (Springer, Berlin, 2000), pp. 365-376.

[24] M. Treiber, A. Hennecke, and D. Helbing, Physical Review E 62, 1805 (2000).

[25] L. Edie and R. Foote, in Highway Research Board Proceedings, 37th annual meeting, edited by $\mathrm{H}$. Orland (National Academy of Sciences, Washington D.C., 1958).

[26] P. de Gennes, Scaling Concepts in Polymer Physics (University Press, Cambridge, 1979).

[27] S. Fedotov and Y. Okuda, Phys. Rev. E 66, 021113 (2002).

[28] M. Schulz, S. Trimper, and B. Schulz, Phys. Rev. E 64, 026104 (2001).

[29] D. Helbing and M. Treiber, Computing in Science and Engineering (CiSE) 5, 89 (1999).

[30] D. Helbing, A. Hennecke, V. Shvetsov, and M. Treiber, Mathematical and Computer Modelling 35, 517 (2002).

[31] B. Kerner, Phys. Rev. Lett. 81, 3797 (1998).

[32] D. Helbing, A. Hennecke, and M. Treiber, Phys. Rev. Lett. 82, 4360 (1999).

[33] P. G. Gipps, Transportation Research B 15, 105 (1981).

[34] M. Treiber, A. Hennecke, and D. Helbing, Phys. Rev. E 59, 239 (1999).

[35] R. Barlovic, L. Santen, A. Schadschneider, and M. Schreckenberg, Traffic and Granular Flow '97 (Springer, Singapore, 1998), p. 335.

[36] M. Treiber and D. Helbing, Cooper@tive Tr@nsport@tion Dyn@mics 1, 3.1 (2002), (Internet Journal, www.TrafficForum.org/journal). 